\title{
Prevalencia e intervención terapéutica en la psicológica clínica privada
}

\section{Prevalence and therapeutic intervention in private clinical psychology}

Fecha de recepción: 27/11/2014

Fecha de aceptación: 23/11/2015

\author{
Abel Baquero Escribano \\ Proyecto Amigó Castellón. Universitat Jaume I \\ Gema Calvo Orenga \\ Grupo Investigación TXP \\ Mar Lluch Quevedo \\ Colegio Oficial Psicólogos Comunidad Valenciana \\ Francisco Santolaya Ochando \\ Colegio Oficial Psicólogos Comunidad Valenciana
}

Gonzalo Haro Cortés

Consorcio Hospitalario Provincial de Castellón. Universidad CEU-Cardenal Herrera, Castellón de la Plana.

\section{resumen/alsstract:}

La intervención terapéutica psicológica desde el ámbito privado se presenta como una de las principales líneas de desempeño laboral en el colectivo de psicólogos, si bien el contenido de esta intervención, y más en concreto la psicopatología en la que se interviene permanece desconocida para el colectivo de psicólogos y para la sociedad en general. Mediante un estudio realizado en la Comunidad Valenciana se obtiene información descriptiva de la actividad clínica privada de un total de 362 psicólogos. Los resultados mostraron una mayor prevalencia de intervención desde la psicología cognitivo conductual. La actividad clínica a nivel privado se relaciona con el tratamiento de trastornos del estado de ánimo y ansiedad en adultos y en trastornos del aprendizaje y problemas de conducta en menores de 16 años.

The psychological therapeutic intervention in the private sphere is presented as one of the main lines of work performance in the group of psychologists, although the content of this intervention, and specifically psychopathology which involved remains unknown for the group of psychologists and society in general. Through a study in the Community of Valencia descriptive data are obtained about descriptive information private clinical activity of a total of 362 psychologists. Results showed a higher prevalence of cognitive behavioral intervention. Clinical privately activity relates to the treatment of mood and anxiety disorders in adults and learning disorders disorders and behavioral problems in children under 16 years

\section{palabras clave/keywords:}

Prevalencia, Intervención Psicológica Privada, Estudio Observacional Transversal.

Prevalence, Psychological Private Intervention, Transversal Observational Study.

La psicología, considerada hasta finales del siglo XIX como una rama de la filosofía, se confirma como una ciencia específica recurriendo al método experimental, a la estadística y a los modelos matemáticos cuando con la fundación en 1879 del laboratorio de Wilhem Wundt en la Universidad de Leipzig se reconoce la labor investigadora de la psicología. La 
transición hacia la vertiente clínica ocurre en 1869 cuando se establece formalmente, por parte de Lighner Witmer, la primera clínica psicológica en la Universidad de Pensilvania para intervenir en las diferencias individuales. Desde ese momento hasta nuestros días la ciencia de la psicología sigue en constante evolución, aparecen nuevas corrientes teóricoprácticas, técnicas e instrumentos de evaluación, configurando progresivamente un mayor protagonismo profesional de los psicólogos en nuestra sociedad. (Rodríguez-Marín 1998).

En España, la historia de la psicología no está claramente delimitada en el tiempo, si bien ya en el Renacimiento aparece el concepto de psicología filosófica atendiendo a humanistas y filósofos como Juan Huarte de San Juan, Juan Luís Vives o Francisco Suárez. Esta línea de pensamiento no tuvo continuidad y fue interrumpida hasta principios del siglo XX, cuando se empieza a tomar conciencia de la importancia de la psicología como ciencia aplicada (Santolaya, Berdullas y Fernández 2002). Es en estos años cuando se produce una progresiva vinculación científica de la psicología en nuestro país, con la creación en 1902 por parte de Luís Simarro del primer laboratorio experimental en la Facultad de Ciencias de la Universidad de Madrid y en 1906, el laboratorio psicofisiológico a cargo de Ramón Turró (Algarabel, 2003), sin embargo la escasa accesibilidad a las fuentes hace difícil precisar el inicio y la evolución de la psicología en España (Carpintero, 1989). En los años 20 aparecen los institutos técnicos en donde los psicólogos aplicados trabajaban con deficientes mentales, enfermos psíquicos y militares. En Madrid se crea el Instituto Nacional de Psicotécnia, en Barcelona el Instituto de Orientación Profesional, cuyo funcionamiento alcanza trascendencia internacional. Con la guerra civil en 1936 se clausuran estos centros, lo que supone una ruptura en el desarrollo y evolución de la psicología en España (Sos, 2011).

Tras la guerra civil, en 1953 se crea la Escuela de Psicología y Psicotecnia en la Universidad Complutense de Madrid, con la finalidad de ofrecer estudios de posgrado en psicología a aquellos licenciados que deseaban la formación en esta área. Éste es considerado el primer centro de formación de psicología en España evolucionando hasta finales de los años sesenta, con la inauguración en la Universidad de Madrid y Barcelona de la Subsección de Psicología dependiente de la sección de Filosofía Pura, con una primera promoción de licenciados en 1972. A nivel clínico, diversas corrientes o escuelas se desarrollan desde que Witmer fundara la primera clínica psicológica. En España, la tradición de la psicología clínica se repartía entre la orientación analítica y la organicista, a mitad de los años 70, la corriente conductual predominaba en la actuación de los profesionales. Las Universidades respondían fundamentalmente al modelo de modificación de conducta, con el tiempo fueron adoptando nuevos paradigmas (Buela-Casal, Sierra, Carrobles, 1995). Desde que se instauró la licenciatura de Psicología en España, el número de licenciados ha ido en vertiginoso aumento pasando de 1.500 en sus inicios a más de 20.000 titulados en los años 80 (Hernández, 1984), en el curso académico 2000-01 el número de matriculados ascendió a 57.000 alumnos. Esta situación hace que, tras la obtención de la titulación y posteriores estudios de especialización, muchos de los profesionales, interesados en el área clínica, intentan desarrollar su intervención el ámbito privado con un mayor número de profesionales en relación al sector público (Palacios, 2004).

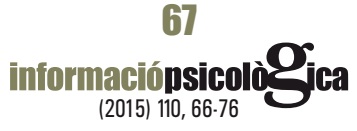


En los últimos años ha habido un aumento en la demanda de atención psicológica, con una mayor prevalencia de cuadros de angustia y estrés. Unido a los cambios y avances en la sociedad, aparecen nuevas demandas terapéuticas relacionadas con una mayor exigencia de la vida, una mayor intolerancia al dolor emocional por parte de los pacientes y una creciente medicalización de la vida cotidiana. (Belloso y Espín, 2007, Echeburúa, 2012)

Este proceso de evolución en la atención psicológica profesional, conlleva diferentes estudios sobre las características personales de los profesionales (Hernández Gordillo, 1984), sobre el enfoque de intervención (Bas Ramallo, 1995, Buela-Casal et cols , 2005, Santolaya, Berdullas y Fernández, 2001) o diagnóstico (Echeburúa, 2012, Estupiñá, Labrador, García-Vera, 2012, Martín-Jurado, De la Gándara, Castro, Moreira y Sánchez-Hernández, 2012), pero cuando atendemos a la situación del psicólogo clínico en su práctica privada, en relación a su enfoque terapéutico o prevalencia de casos, no se encuentran estudios sistematizados que faciliten esta información, si bien existen aproximaciones (Borkobev, 2004, Stirman, DeRubeis, Crits-Christoph y Rothman, 2005).

A pesar de los esfuerzos por obtener un conocimiento más preciso de la práctica asistencial privada de la psicología clínica, todavía quedan algunas incógnitas, sobre todo en lo que se refiere a cuestiones relativas al tipo de trastornos por el que se demanda más atención terapéutica, los tratamientos disponibles, los utilizados y los resultados obtenidos con cada uno de ellos. En este sentido este estudio pretende describir la labor que realizan los psicólogos de práctica clínica en el ámbito privado mediante un análisis descriptivo de los datos obtenidos y comparándolos con los existentes el sistema público de salud mental.

\section{Método.}

\section{Participantes}

Mediante un proceso de encuesta online y un proceso de selección probabilístico aleatorio se contacta con el total de psicólogos colegiados en la Comunidad Valenciana. De los sujetos contactados un total de 587 contestan la encuesta, tras la revisión de las mismas se encuentran errores a nivel de selección muestral, principalmente el no desempeñar actividad clínica privada 161 sujetos y los restantes 64 sujetos por problemas en la cumplimentación de la encuesta configurándose el tamaño muestral definitivo en 362 sujetos.

\section{Instrumentos de recogida de datos.}

Para la recogida de datos en este estudio descriptivo se utilizó una encuesta estructurada de 9 ítems de selección múltiple definidos en dos áreas, una centrada en el perfil clínico del psicólogo que cumplimenta la encuesta, donde se recoge la ubicación geográfica laboral asî como el enfoque de intervención y el otro centrado en los datos objetivos de la intervención clínica privada en donde se detalla el número de pacientes atendidos, sexo, edad y diagnóstico clínico DSM- IV-R.

El plazo de tiempo que se estableció para la recogida de la muestra fue durante el año 2013, recordando trimestralmente los objetivos y procedimiento del estudio de la investigación en vistas a la selección de sujetos. 


\section{Procedimiento.}

Una vez se contacta con los participantes, éstos contestan a la encuesta, de acuerdo a los criterios previamente indicados. Una vez cumplimentadas las encuestas se transferían los datos en un recurso web diseñado para este procedimiento. La administración de estas encuestas se realizó siempre de forma individual y siguiendo los preceptos establecidos por la APA (American Psychological Association), (APA, 2011). Las pruebas fueron diseñadas y analizadas por profesionales del ámbito de la psicología formados en el diseño e implementación de la investigación.

Los datos obtenidos durante la evaluación de los sujetos se codificaron mediante un procedimiento de encriptación para asegurar la confidencialidad. El análisis estadístico comenzó por una exploración descriptiva de las variables seleccionadas para este trabajo, para ello se utilizó el programa estadístico SPSS 21.

\section{Resultados.}

En la distribución de los encuestados según ubicación geográfica obtiene un mayor tamaño en la provincia de Valencia, seguida de Alicante y Castellón, parece obvio ya que el número de colegiados sigue también esa distribución (ver tabla 1).

Tabla 1. Distribución de los encuestados según ubicación geográfica.

\begin{tabular}{|ccc|}
\hline & $n$ & Porcentaje \\
\hline Alicante & 111 & 30.66 \\
Castellón & 52 & 14.36 \\
Valencia & 199 & 54.98 \\
Total & 362 & 100 \\
\hline
\end{tabular}

Tabla 2. Distribución según escuela metodológica.

\begin{tabular}{lcc|} 
& $n$ & Porcentaje \\
\hline Cognitivo-Conductual & 249 & 68.78 \\
Psicoanálisis & 48 & 13.26 \\
Sistémica & 25 & 6.90 \\
Dialéctico-Conductual & 8 & 2.22 \\
Gestalt & 8 & 2.22 \\
Humanista & 8 & 2.22 \\
Neuropsicología & 8 & 2.22 \\
EMDR & 3 & .83 \\
Existencialista/ Logoterapia & 1 & .27 \\
Conductual & 1 & .27 \\
Programación neurolingüística & 1 & .27 \\
Psicocorporal & 1 & .27 \\
Transpersonal & 1 & .27 \\
\hline Total & 362 & 100 \\
\hline
\end{tabular}


En relación al enfoque laboral, y escuela metodológica de intervención (ver tabla 2), la totalidad de los encuestados desarrollaba su actividad asistencial dentro de la psicología clínica, con una predominancia de intervención desde el modelo cognitivo-conductual con un $68.78 \%$ de los participantes.

\section{Variables sociales de los pacientes atendidos.}

El número de pacientes atendidos asciende a un total de 7.517 y la distribución por provincias obtiene un mayor número de pacientes en la provincia de Valencia con 4.332, seguida de Alicante con 2.338 y Castellón con 847 pacientes atendidos, lo cual se explicaría atendiendo a la distribución poblacional pero también a la distribución de encuestados (tabla 1). La distribución por sexo de los pacientes atendidos se configura en $49.45 \%$ hombres y $51.55 \%$ mujeres. Otro dato que también guarda estrecha relación con el tipo de actividad clínica es la edad del paciente (Norcross, Knight, 2000), ésta se distribuye en $14.15 \%$ de pacientes con una edad comprendida entre 16 y 24 años, un $49.05 \%$ de pacientes de 25 a 44 años, un $28.17 \%$ de 45 a 64 años, un $7.53 \%$ de 65 a 74 años, un $1.11 \%$ de 75 a 84 años y un $1.19 \%$ mayores de 85 años. Por tanto, el intervalo de edad en el que se detecta un mayor porcentaje de pacientes es de 25 a 44 años. Los datos más específicos en relación a la variable edad se detallan en tabla 3 (Ver tabla 3).

Tabla 3. Distribución por provincia de los pacientes atendidos según edad.

\begin{tabular}{|c|c|c|c|c|c|c|c|c|}
\hline & $\begin{array}{c}\text { Casos } \\
<16 \text { años }\end{array}$ & $\begin{aligned} & \text { Casos } \\
> & 16 \text { años }\end{aligned}$ & $\begin{array}{c}16-24 \\
\text { años }\end{array}$ & $\begin{array}{c}25-44 \\
\text { años }\end{array}$ & $\begin{array}{c}45-64 \\
\text { años }\end{array}$ & $\begin{array}{c}65-74 \\
\text { años }\end{array}$ & $\begin{array}{l}>75 \\
\text { años }\end{array}$ & Total \\
\hline Valencia & 964 & 3.368 & 10.6 & 47.4 & 31.7 & 9.1 & $1.2^{*}$ & 100 \\
\hline Castellón & 116 & 731 & 26.6 & 53.7 & 17.1 & 1.7 & 0.9 & 100 \\
\hline Alicante & 357 & 1.981 & 23.9 & 54.3 & 18.8 & 2.3 & 0.7 & 100 \\
\hline$n$ & 1.437 & 6.080 & & & & & & \\
\hline
\end{tabular}

Cuando se detallan los casos atendidos en consulta privada, diferenciamos dos categorías de trastornos y pacientes atendidos: adultos y diagnósticos en casos infanto-juveniles, es decir pacientes menores de 16 años. La distribución de los casos por provincia se detalla en la tabla 3, donde se evidencia un mayor número de casos menores de 16 años en la provincia de Valencia en relación con Alicante y Castellón, esta distribución parece seguir en adultos una distribución acorde con la distribución poblacional. Cuando se analizan las diferencias de proporciones mediante la prueba $Z$, se obtiene que el porcentaje de sujetos con edades comprendidas entre los 45 y 74 años es estadísticamente mayor en la provincia de Valencia*, a la vez que existen diferencias estadísticamente significativas entre provincias $\left(X^{2}=\right.$ $53.3 p<.05)$ 
La distribución de los casos por categoría diagnóstica en mayores de 16 años se configura con una mayor prevalencia en la atención de trastornos del estado de ánimo (25.02\%) seguida de trastornos de ansiedad (18.24\%) y trastornos adaptativos, el resto de categorías obtienen valores promedio entre el (1.63 y el 5.17\%). (Ver tabla 4). En relación a la variable sexo existe una mayor prevalencia de mujeres en trastornos del estado de ánimo (67.3\%) y trastornos de ansiedad (59.4\%).

Tabla 4. Distribución de los pacientes adultos atendidos según categoría diagnóstica en la Comunidad Valenciana.

\begin{tabular}{|lcccc|}
\hline & Valencia & Castellón & Alicante & Total \\
Trastornos del estado de ánimo & 24.45 & 21.95 & $28.68^{*}$ & 25.02 \\
Déficit o retraso mental & 1.52 & 1.13 & 2.25 & 1.63 \\
Trastornos alimentarios & 5.84 & 2.93 & 6.76 & 5.17 \\
Trastornos adaptativos & 16.35 & 23.55 & 14.26 & 18.05 \\
Trastornos por uso de sustancias & 4.71 & 1.13 & 3.36 & 3.06 \\
Tabaquismo & 2.45 & 1.13 & 6.78 & 3.45 \\
Trastornos ansiedad & 16.76 & $23.66^{*}$ & 14.30 & 18.24 \\
Trastornos psicóticos & 2.99 & 1.13 & 5.24 & 3.12 \\
Trastornos sexuales & 1.54 & 2.37 & 3.91 & 2.60 \\
Trastornos personalidad cluster A & 2.34 & 1.23 & 1.80 & 1.79 \\
Trastornos personalidad cluster B & 5.56 & 2.75 & 3.30 & 3.87 \\
Trastornos personalidad cluster C & 2.22 & 1.45 & 2.87 & 2.18 \\
Otros trastornos & 13.27 & 15.59 & 6.49 & 11.82 \\
Total & 100 & 100 & 100 & 100 \\
\hline
\end{tabular}

Cuando se analizan las categorías diagnósticas según provincias, encontramos valores idénticos a los reflejados en el global de la Comunidad Valenciana, con valores más elevados en trastornos del estado de ánimo en la provincia de Alicante (28.68\%), y trastornos adaptativos y de ansiedad en Castellón con valores de $23.55 \%$ y $23.66 \%$ respectivamente (ver tabla 4). Cuando se analizan las diferencias de proporciones mediante la prueba $Z$, se obtiene que el porcentaje de sujetos con trastornos del estado de ánimo es superior en la provincia de Alicante* en relación a Valencia, así como el porcentaje de trastornos de ansiedad es superior en Castellón*, a la vez que existen diferencias estadísticamente significativas entre provincias $\left(X^{2}=44.9 p<.000\right)$

La actividad asistencial privada en menores de 16 años, se centra principalmente en trastornos del aprendizaje $27.80 \%$, problemas de conducta $16.95 \%$, trastornos de la comunicación 
$12.58 \%$ y déficit o retraso mental con un $10.56 \%$, en resto de categorías diagnosticas oscilan entre el $1.90 \%$ del trastorno de tics y el $10.85 \%$ de otros trastornos (Ver tabla 5). Respecto a la distribución por sexo de los pacientes, existe una mayor prevalencia de sujetos de sexo masculino en los anteriores trastornos con un $74 \%$ de varones en trastornos del aprendizaje, $61.9 \%$ en problemas de conducta y un $73 \%$ en trastornos de la comunicación.

Tabla 5. Distribución de los pacientes menores de 16 años atendidos según categoría diagnóstica en la Comunidad Valenciana.

\begin{tabular}{|lcccc|}
\hline & Valencia & Castellón & Alicante & Total \\
Déficit o retraso mental & $14.54^{*}$ & 6.63 & 10.52 & 10.56 \\
Problemas de conducta (Incluye TDAH) & 12.74 & 21.14 & 16.96 & 16.95 \\
Trastornos estado ánimo & 4.25 & 2.37 & 5.84 & 4.15 \\
Trastornos ansiedad & 8.70 & 4.76 & 4.15 & 5.87 \\
Trastornos aprendizaje & 26.65 & 33.92 & 22.83 & 27.80 \\
Trastornos eliminación & 2.10 & 0 & 4.45 & 2.18 \\
Trastornos generalizados del desarrollo & 4.28 & 7.67 & 9.54 & 7.16 \\
Trastornos tics & 3.37 & 0 & 2.34 & 1.90 \\
Trastornos comunicación & 11.12 & 17.28 & 9.35 & 12.58 \\
Otros trastornos & 12.25 & 6.23 & 14.02 & 10.85 \\
Total & 100 & 100 & 100 & 100 \\
\hline
\end{tabular}

$\mathrm{Al}$ analizar las categorías diagnósticas según provincias (ver tabla 5), aparecen una distribución más heterogénea que la obtenida en la Comunidad Valenciana, con categorías incluso que no registran casos, como trastornos de la eliminación con una prevalencia de $2.10 \%$ en Valencia, $4.45 \%$ en Alicante y ningún caso en Castellón o trastornos por tics, sin registrar pacientes en Castellón y con $3.37 \%$ en Valencia y $2.34 \%$ en Alicante. Por otro lado, y pese a presentar categorías diagnósticas sin pacientes, la provincia de Castellón obtiene el mayor porcentaje de pacientes atendidos en trastornos del aprendizaje con un $33.92 \%$ de pacientes menores de 16 años atendidos. Al analizar las diferencias de proporciones mediante la prueba $Z$, se obtiene que el porcentaje de sujetos con déficit o retraso mental es estadísticamente mayor en la provincia de Valencia*.

\section{Discusión.}

Este estudio describe la actividad psicológica clínica ejercida en el ámbito privado y las características de esta atención en la Comunidad Valenciana. Nuestros resultados concluyen que el paradigma cognitivo-conductual es el que predomina en la intervención asistencial privada en la Comunidad Valenciana, esto seguiría la línea de diferentes estudios (Bas, 1995, Santolaya, Berdullas y Fernández, 2001). 
Las características sociales de los pacientes condicionan en gran medida el tipo de asistencia dispensada, en relación a la edad de los sujetos atendidos en consulta privada esta se sitúa en una mayor prevalencia de atención psicológica clínica entre los 25 y 44 años de edad, tanto para hombres como mujeres. En diferentes estudios la edad de los pacientes en la atención pública en salud mental difiere de los datos obtenidos con una mayor atención en edades de entre 35 a 54 años (Bartoll, Palència, Malmusi, Suhrcke y Borrell, 2013) o 50 a 64 años (Haro y cols. 2006).

En relación al sexo de los pacientes existe un mayor porcentaje de mujeres que reciben asistencia psicológica privada, al analizar los datos se detecta un mayor porcentaje de mujeres en las categorías clínicas de mayor demanda de tratamiento: trastornos del estado de ánimo y de ansiedad, este dato se corresponde en la asistencia pública con también una mayor atención a mujeres en estos trastornos tanto a nivel nacional, (Alonso y cols. 2004), como internacional (Kessler, 2006, Seedat y cols. 2009, Kravitz, Schott, Joffe, Cyranowski y Bromberger, 2012, Olfson, Marcus, Druss, Elinson, Tanielian y Pincus, 2002). No obstante los porcentajes varían en función de diferentes investigaciones, por otro lado en relación también al sexo del paciente, existen estudios que apuntan a un incremento del porcentaje de varones atendidos en los servicios de atención mental pública debido a la crisis económica como estresor exógeno (Bartoll, Palència, Malmusi, Suhrcke y Borrell, 2013) o más en concreto como en la Encuesta de La Salud de la Comunitat Valenciana 2010, donde aparece una mayor prevalencia de varones con trastornos de tipo depresivo. En relación al diagnóstico del paciente, no todos los datos son idénticos a los obtenidos, diversas investigaciones encuentran que la mayor prevalencia se produce en trastornos de ansiedad en lugar de los relacionados con el estado de ánimo (Kessler, Chiu, Demler y Walters, 2005). Independiente de la variable sexo, aparece un aumento en la prevalencia y tratamiento de estos trastornos debido a la influencia de la situación económica actual y sus consecuencias a nivel de estresor social: deprivación económica, etc. (Gili, Roca, Basu, McKee, y Stuckler, 2012).

En lo referente a pacientes menores de edad, se observa una inversión de resultados en torno a la variable sexo, con mayor número de sujetos masculinos menores de 16 años, donde se halla una mayor prevalencia en nuestros resultados de trastornos del aprendizaje con un $27.80 \%$ de los menores atendidos clínicamente a nivel privado. Existen estudios a nivel nacional e internacional en atención pública que obtienen valores similares a los de este estudio (Feightner, 1994, Taylor, 1989). Los trastornos de conducta ocupan el segundo lugar con un $16.95 \%$, un porcentaje inferior frente al de diferentes estudios (Aláez, Martínez, Rodriguez, 2000), estos trastornos ocuparían también el segundo lugar, en el Encuesta de La Salud de la Comunitat Valenciana 2010, como en nuestros datos se observa una elevada atención a trastornos de conducta (incluyendo TDAH) aunque cabe comentar que en este último estudio como en otros aparece una elevada prevalencia de pacientes con retraso mental (Criado y Romo, 2003, WHO, 2007), este diagnóstico se sitúa en quinto lugar en nuestro estudio con valores del $10.56 \%$, si bien existe una enorme variabilidad en los datos de prevalencia sobre estos trastornos, situando por ejemplo una prevalencia estimada del retraso mental del $0.5 \%$ en Cataluña (García-Ibañez, y col. 2003), 1,4\% en Australia (Leonard, Petterson, Bower y Sanders, 2003) y del 0.6\% en Irlanda (McConkey, Mulvany 
y Barron, 2006). En relación a los trastornos de la comunicación, ocupan el tercer lugar con un $12.58 \%$, los datos de prevalencia en la asistencia clínica pública se sitúan en torno al 5-10\% (Ruben, 2000). Parece que a diferencia de los datos relativos a la prevalencia de trastornos psicológicos en adultos, los indicadores de prevalencia obtenidos en la asistencia clínica infanto-juvenil privada, difieren notablemente en función de los diferentes resultados existentes.

Cuando analizamos las principales características de la atención psicológica clínica privada en relación al perfil profesional del terapeuta y los aspectos relacionados con la prevalencia de trastornos mentales en adultos, se detecta un paralelismo, que no se da en menores de 16 años. Parece pues que con los datos que se obtienen en el estudio, se confirme una duplicidad asistencial en las principales categorías diagnósticas respecto a la red asistencial privada y pública, si bien puede que los motivos de esta similitud en la atención clínica, aunque no existen apenas estudios al respecto, se relacione con una insuficiente cobertura asistencial pública que condicione la atención privada, tiempos de atención más dilatados, aspectos relativos a la privacidad, etc. como referimos anteriormente este es un tema en el que no existe apenas información disponible, por lo que consideramos relevantes los datos que aporta nuestro estudio, siendo conscientes de las limitaciones a nivel estadístico y del tamaño muestral, ya que pese al número de colegiados en la Comunidad Valenciana cerca de 6.700, tan sólo participan en este estudio 362 , habiendo sido informados la totalidad de los colegiados.

Múltiples aspectos faltan aún por conocer de la atención psicológica privada, más aún si cabe con la inminente regularización de los psicólogos como profesionales sanitarios y la actividad de los mismos. Este es un campo de estudio que debe progresivamente adquirir mayor relevancia, no sólo por su función epidemiológica, y científica sino también por la consecuente relación con la calidad y adecuación de la actividad psicológica a nivel pública, también a día de hoy en un proceso de cambio y reconversión.

\section{Referencias}

Aláez, M., Martínez, R. Rodríguez, C. (2000). Prevalencia de trastornos psicológicos en niños y adolescentes, su relación con la edad y género. Psicothema, 4, 525,532.

Algarabel, S., Luciano, J.V. (2003). Psychology in Spain. The Psychologist vol. $16 \mathrm{n}^{0} 4$.

Alonso. J., Angermeyer. M.C., Bernert. S., Bruffaerts. R., Brugha. T.S., Bryson. H., y cols. (2004). Prevalence of mental disorders in Europe: Results from the European Study of the Epidemiology of Mental Disorders (ESEMeD) Project. Acta Psychiatrica Scandinavica, $109(420), 21-27$.

American Psychological Association (APA, 2011). Publication Manual of the American Psychological Association (6 ${ }^{\circ}$ ed.) (pp. 13-16). Washington, DC: American Psychological Association.

Bartoll, X., Palència, L., Malmusi. D., Suhrcke., M. Borrell, C. (2013). The evolution of mental health in Spain during the economic crisis. European Journal of Public Health, doi:10.1093/eurpub/ckt208

Bas, F. (1995). Reflexiones sobre la psicología clínica en España y sobre la acreditación del psicólogo clínico. Psicología Conductual, 3, 401. 418. 
Belloso, J.J. y Espín, J.C. (2007). Un año de pacientes nuevos (A year of new patients). Revista Española de Neuropsiquiatría, 27(99), 39-58.

Borkovec, T. D. (2004). Research in training clinics and practice research networks: A route to the integration of science and practice. Clinical Psychology: Science and Practice, 11, 212-216.

Buela-Casal, G., Bretón-López, J., Agudelo, D., Bermúdez, M.P., Sierra, J. C., Teva, I. y Gil-Roales-Nieto, J. (2005). Imagen de la psicología como profesión sanitaria en psicólogos españoles. Papeles del psicólogo, 26, 16-23.

Buela-Casal G., Sierra J.C., Carrobles J.A. (1995). Psicología clínica y de la salud en España, su estado actual. Revista latinoamericana de psicología, 27(1), 25-40.

Carpintero, H. (1989). El psicólogo en España. Notas históricas sobre su desarrollo profesional. Papeles del psicólogo, 36/37, 68-73.

Criado, J.J. y Romo. C. (2003). Variabilidad y tendencias en el consumo de metilfenidato en España. Estimación de la prevalencia del trastorno por déficit de atención con hiperactividad, Revista de Neurología, 37, 806-810.

Echeburúa, E., Salaberría, K., De Corral, P., Cruz-Sáez, S. (2012). Funciones y ámbitos de actuación del psicólogo clínico y del psicólogo general sanitario: una primera reflexión. Behavioral Psychology / Psicología Conductual, 20(2), 423-435.

Encuesta de La Salud de la Comunitat Valenciana 2010. (2012). Generalitat, Conselleria de Sanitat (Eds). Valencia, 2012.

Estupiñá, F., Labrador, F. J., García-Vera, M. P. (2012). A study of patients who go to a psychology clinic seeking treatment. The Spanish Journal of Psychology, 15(1), 275-285.

Feightner, J.W. (1994). Preschool screening for developmental problems. En: Canadian task force on the periodic health examination. Canadian guide to clinical preventive health care. Ottawa: Ottawa Health Canada, 1994

García Ibáñez, J., Camprubí, A., Almenar, C., Angel, S., Bouzas, X., Cardona, L. y cols. (2003). Necesidades de salud mental en personas con discapacidad intelectual (SM-DI), Quaderns de Salut Menta/ $\mathrm{n}^{0}$ 5. Barcelona: CatSalut.

Gili, M., Roca, M. M., Basu, S., McKee, M. Stuckler, D. (2012). The mental health risks of economic crisis in Spain: evidence from primary care centers, 2006 and 2010. The European Journal of Public Health, 23. 103-108.

Haro, J. M., Palacín, C., Vilagut, G., Martínez, M., Bernal, M., Luque, l., et cols (2006). Prevalencia de los trastornos mentales y factores asociados: resultados del estudio ESEMeD-España [Prevalence of mental disorders and associated factors: results from the ESEMeD. Spain study]. Medicina Clinica, 126, 445-451.

Hernández, A. (1984). La psicología como profesión. Papeles del psicólogo, 6/17, 61-63.

Kessler, R.C., Chiu, W.T, Demler. O, y Walters. E.E. (2005). Prevalence, severity, and comorbidity of twelve-month DSM-IV disorders in the National Comorbidity Survey Replication (NCS-R). Archives of General Psychiatry, 62(6),617-27.

Kessler, R.C. (2006). The epidemiology of depression among women. In C. Keyes \& S. Goodman (Eds.), A Handbook for the Social, Behavioral, and Biomedical Sciences: Women and Depression (pp. 22-37). New York: Cambridge University Press.

Kravitz. H.M., Schott, L.L., Joffe, H., Cyranowski, J. M. y Bromberger, J.T. (2012), Do anxiety symptoms predict major depressive disorder in midlife women? The Study of Women's Health Across the Nation (SWAN) Mental Health Study (MHS). Psychological Medicine, 27, 1-10.

Leonard. .H, Petterson. B., Bower. C., Sanders. R. (2003). Prevalence of intellectual disability in Western Australia. Paediatric and Perinatal Epidemiology, 17,58-67.

Martín-Jurado, A., De la Gándara, J.J., Castro, S., Moreira, A., Sánchez-Hernández, J. (2012). Análisis de la concordancia de las derivaciones de atención primaria a Salud Mental. Semergen, 38(6) 354-359.

McConkey, R., Mulvany, F., Barron, S. (2006). Adult persons with intellectual disabilities on the island of Ireland. Journal of Intellectual Disability Research, 50, 227-236.

Norcross, J., Knight, B. Psychotherapy and aging in the 21 st century: Integrative themes. In Qualls \& Abeles (eds). Psychology and the aging revolution: How we adapt to longer life. Washington, DC, US: American Psychological Association, 2000. doi: 10.1037/10363-014 
Olfson, M, Marcus, SC, Druss B, Elinson L, Tanielian T, Pincus H.A. (2002). National trends in the outpatient treatment of depression. Journal of the American Medical Association, 287, 203-209

Palacios, A. J. (2004). Sobre la profesión de psicólogo clínico: Consideraciones actuales y retos futuros. Revista de la Asociación Española de Neuropsiquiatría, 91,139-147.

Rodríguez-Marín, J. (1998). Psicología de la salud y psicología clínica. Papeles del psicólogo, 69.

Ruben, R. (2000). Redefining the survival of the fittest: Communication disorders in the21st century. Laryngoscope, 110(2), 241-245

Santolaya, F., Berdullas, M. y Fernández, J.R. (2001). The decade 1989-1998 in Spanish Psychology: An analysis of development of Development of Professional Psychology in Spain. The Spanish Journal of Psychology, 4, 237-252.

Seedat. S., Scott, K., Angermeyer, M., Berglung, P., Bromet, E., Brugha, T. y cols. (2009). Cross-national associations between gender and mental disorders in the World Health Organization World Mental Health Surveys. Archives of General Psychiatry, 66, 785-795.

Sos R., Calatayud C. (2011). La psicología aplicada española en tiempos de posguerra (1939-1945). Revista de Historia de la Psicología, $32,2$.

Stirman, S.W., DeRubeis, R.J., Crits-Christoph, P., Rothman, A. (2005). Can the randomized controlled trial literature generalize to non randomized patients? Journal of Consulting and Clinical Psychology, 73, 127-135.

Taylor, H.G. Learning disabilities. In Marsh \& Barkley (eds). Treating the childhood disorders. New York: Guildford, 1989.

World Health Organization (WHO). 2007. Atlas: global resources for persons with intellectual disabilities. Geneva. World Health Organization 